\title{
Intervenções em competências sociais com adolescentes e o desenvolvimento de atitudes positivas em situação de vulnerabilidade: uma revisão sistemática
}

\author{
Rafael Corrêa, MS $1,2,3$ \\ Loris Prado da Cruz, $\mathrm{Ms}^{2}$ \\ Diego Gomez-Baya, $\mathrm{PhD}^{4}$ \\ Ana Maria de Almeida, $\mathrm{PhD}^{2}$ \\ Margarida Gaspar de Matos, $\mathrm{PhD}^{3}$ \\ 1 Universidade Estadual do Oeste do Paraná, Brasil. \\ 2 Universidade de São Paulo, Brasil \\ ${ }^{3}$ Universidade de Lisboa, Portugal \\ ${ }^{4}$ Universidad de Huelva, España
}

RESUMO

O objectivo deste trabalho foi avaliar evidências científicas oriundas de estudos de intervenções em competências sociais com adolescentes para o desenvolvimento de atitudes positivas em situação de vulnerabilidade. Os estudos foram identificados a partir de buscas nas bases de dados LILACS, PubMed, PsycINFO e SCOPUS no período de 2008 até 2018. Os critérios de selecção foram publicações em inglês, português ou espanhol, estudos primários de intervenção em competências sociais e participantes adolescentes. A revisão incluiu 17 estudos relacionados a intervenções em competências sociais utilizando como estratégias instruções e actividades semanais, instruções e grupos com interacção social, somente instruções e sistema mobile coach. As intervenções em competências sociais com adolescentes em situação de vulnerabilidade são estratégias eficazes para o desenvolvimento de atitudes positivas. Estes resultados fornecem um guia baseado em evidências para investigadores, profissionais e instituições para a prática em prevenção e promoção a saúde de adolescentes.

PalaVRas-ChaVe

Adolescência; saúde; vulnerabilidade; competências sociais.

\section{RESUMEN}

El objetivo de este trabajo fue evaluar la evidencia científica de estudios de intervenciones en competencias sociales con adolescentes para desarrollar actitudes positivas en situaciones vulnerables. Los estudios se identificaron a partir de búsquedas en las bases de datos LILACS, PubMed, PsycINFO y SCOPUS de 2008 a 2018. Los criterios de selección fueron: publicaciones en inglés, portugués o español; estudios primarios de intervención en competencias sociales; y participantes adolescentes. La revisión incluyó 17 estudios relacionados con intervenciones de competencias sociales que usaron instrucción y actividades semanales, grupos de instrucción e interacción social, sólo instrucción, y entrenamiento mediante teléfono móvil, como estrategias. Las intervenciones de competencias sociales con adolescentes vulnerables son estrategias efectivas para desarrollar actitudes positivas. Estos hallazgos proporcionan una guía basada en evidencia para investigadores, profesionales e instituciones para la práctica en la prevención y promoción de la salud de los adolescentes.

PaLABRAs CLAVE

Adolescencia; salud vulnerabilidad; competencias sociales.

O presente trabalho foi realizado com apoio da Coordenação de Aperfeiçoamento de Pessoal de Nível Superior - Brasil (Capes) - Código de Financiamento 001

Recibido: 04/03/2020; aceptado: 28/05/2020.

Correspondencia: Rafael Corrêa, Faculdade de Motricidade Humana, Universidade de Lisboa, Estrada da Costa, 1499-002 Cruz Quebrada, Portugal. Telefone: (+351) 214149152, E-mail: rs.correa@hotmail.com 
A questão da vulnerabilidade em saúde de adolescentes tem sido um tema abordado por diversas áreas de actuação como uma questão problema que exige esforços de gestores, profissionais pesquisadores no desenvolvimento de intervenções eficazes. Dentre as questões mais prevalentes que envolvem a população adolescentes destaca-se sexualidade, o uso de álcool e drogas e a delinquência juvenil (Botvin \& Griffin, 2014).

Estudos identificaram que os programas de promoção de competências sociais e de vida realizado em ambiente escolar são eficazes para a prevenção do uso de substâncias, depressão, delinquência juvenil, melhora no bem estar de adolescentes fortalecendo suas atitudes para lidar com questões do quotidiano. Entretanto, a disseminação de tais programas, muitas vezes, são dificultados pela disponibilidade de pessoal, questões de financiamento, definição e metodologia da abordagem (Haug et al., 2017). As competências sociais são parte do núcleo das interacções diárias de cada indivíduo desde a infância até a fase adulta, e através de classes de comportamentos aprendidas, em situações específicas, permitem ao indivíduo ser aceite ou evitar comportamentos inaceitáveis socialmente (Beauchcamp \& Anderson, 2010). Sofrem influencia de factores individuais, contextuais e culturais e apresentam dificuldade na definição e, consequentemente, em intervenções devido a ampla variedade teórica.

O deficit de competências sociais é considerado factor de risco, sendo assim a melhora nas interacções sociais seria o principal objectivo das intervenções. Deste modo, programas visando a prevenção e a promoção em saúde com o desenvolvimento de atitudes positivas em adolescentes têm sido foco de intervenção recente para essa abordagem (Eslami et al., 2015; Haug et al., 2017; Matos et al., 2012; Roberts et al., 2011; van der Stouwe, Asscher, Hoeve, van der Laan, \& Stams, 2018).

Alguns modelos de programas de intervenção em competências sociais com adolescentes, são apontados como eficazes para prevenção de comportamento de risco e promoção de comportamentos de protecção. Porém o formato e a influência de factores externos para a generalização e manutenção dos comportamentos aprendidos têm sido questionados (Babakhani 2011; Kimber \& Sandell, 2009; Roberts et al., 2011). Algumas abordagens provenientes da terapia cognitivo comportamental, abordagens multicomponentes, uso de ferramentas electrónicas, estão surgindo como opção eficaz para desenvolvimento de atitudes positivas (Bonsergent et al., 2013; Haug et al., 2017). Deste modo, este estudo visa avaliar evidências científicas oriundas de estudos de intervenções em competências sociais com adolescentes para o desenvolvimento de atitudes positivas em situação de vulnerabilidade.

\section{Método}

Esta revisão sistemática foi realizada e registada de acordo com as directrizes do Preferred reporting items for systematic review and metaanalysis protocols (PRISMA-P) (Moher et al., 2015). Os conceitos principais em estudo medidos foram intervenções em competências sociais com adolescentes e o desenvolvimento de atitudes positivas em situação de vulnerabilidade. 


\section{Critério de inclusão}

Os artigos de fonte primária relacionados à eficácia da intervenção em competências sociais publicados no período de janeiro de 2008 a dezembro de 2018 foram elegíveis para inclusão no estudo. Para tal foram considerados critérios de elegibilidade: (1) desenho de estudo empírico de intervenção (critério de desenho do estudo), (2) estudos de intervenção em competências sociais e o desenvolvimento de atitudes positivas (critério de resultado); adolescentes de 12 a 20 anos de idade em situação de vulnerabilidade (critério de participantes), (4) artigos publicados em inglês, português ou espanhol (critério de linguagem).

\section{Estratégia de busca e selecção de estudos}

Os estudos foram identificados em busca nas seguintes bases de dados electrónicas: LILACS, PubMed, PsycINFO e SCOPUS. Para a identificação dos artigos utilizaram-se os termos: ("Social Skills" OR "Social Skill" OR "Social, Abilities" OR "Social Ability" OR "Interpersonal Skills" OR "Interpersonal Skill" OR "Social Competence") AND (Adolescent OR Adolescents OR Adolescense OR Youth NOT Autism OR Child OR Children OR Neonate* OR Pediatric*).

Títulos obtidos e resumos foram avaliados independentemente para elegibilidade para inclusão por dois autores. Entradas duplicadas foram removidas. Artigos que atendiam aos critérios de inclusão, pela leitura do título e resumo foram recuperados para uma leitura completa. As referências desses artigos foram pesquisadas para encontrar quaisquer outros estudos relevantes Os mesmos dois autores revisaram o texto de estudos potenciais e as decisões de incluir ou excluir estudos na revisão foram feitas por consenso e análise de uma terceira pessoa.

\section{Extracção e harmonização de dados}

Um formulário de extracção de dados foi desenvolvido com base na declaração PRISMA (Moher et al., 2015). Dados relevantes foram extraídos de manuscritos por um autor e a codificação foi verificada por dois autores. Desacordos foram resolvidos por discussão entre os autores. Os dados extraídos incluíram autor, ano e país; tipo de estudo, amostra; instrumentos; intervenção, abordagem teórica; qualidade do estudo e resultados.

\section{Análise do estudo, qualidade e risco de viés}

A qualidade do estudo foi avaliada usando a lista de verificação da Avaliação Crítica do EBL (Glynn, 2006). Esta ferramenta de avaliação de validade, aplicabilidade e relevância dos estudos incluídos com base em quatro domínios de pesquisa: população, colecta de dados, desenho do estudo e resultados. A validade global (classificação global) dos estudos foi determinada com base nos escores "Sim" $\geq 75 \%$ ou nos escores "Não/Pouco claro" $\leq 25 \%$. Dois pesquisadores classificaram os artigos, as discrepâncias foram resolvidas por concordância.

\section{Resultados}

\section{Pesquisa bibliográfica}

O fluxograma dos estudos incluídos nesta revisão sistemática é apresentado na Figura 1. Na pesquisa sistemática nas bases de dados identificaram-se 2.097 registos, desses, 964 resumos 
foram avaliados para elegibilidade após exclusão de duplicatas ( $n=1.133)$. Um total de 926 artigos foram rejeitados após o título e o resumo serem lidos. Posteriormente, 38 artigos potencialmente relevantes em texto completo foram avaliados, com um total de 17 artigos incluídos.

\section{Características dos estudos incluídos}

A tabela 1 apresenta as características dos artigos incluídos, sendo que o desenho do estudo mais comum foi o quase-experimental pré e pós-teste $(n=5)$ seguido de ensaio clínico de controlo randomizado $(n=3)$. Com relação a população adolescente em condições de vul-

\section{Principais resultados}

Instruções e actividades semanais. A estratégia instruções e actividades semanais foi relacionada com programas com encontros semanais de grupo visando fornecer informações sobre competências sociais, temas de saúde ou cidadania e a indicação da realização de actividades como tarefas de casa, exercícios físicos, acompanhamento multiprofissional, técnicas cognitivo comportamentais e alguns estudos foram desenvolvidos com componente parental (Tabela 3). A estratégia de instruções e actividades semanais em sua grande maioria, bus-

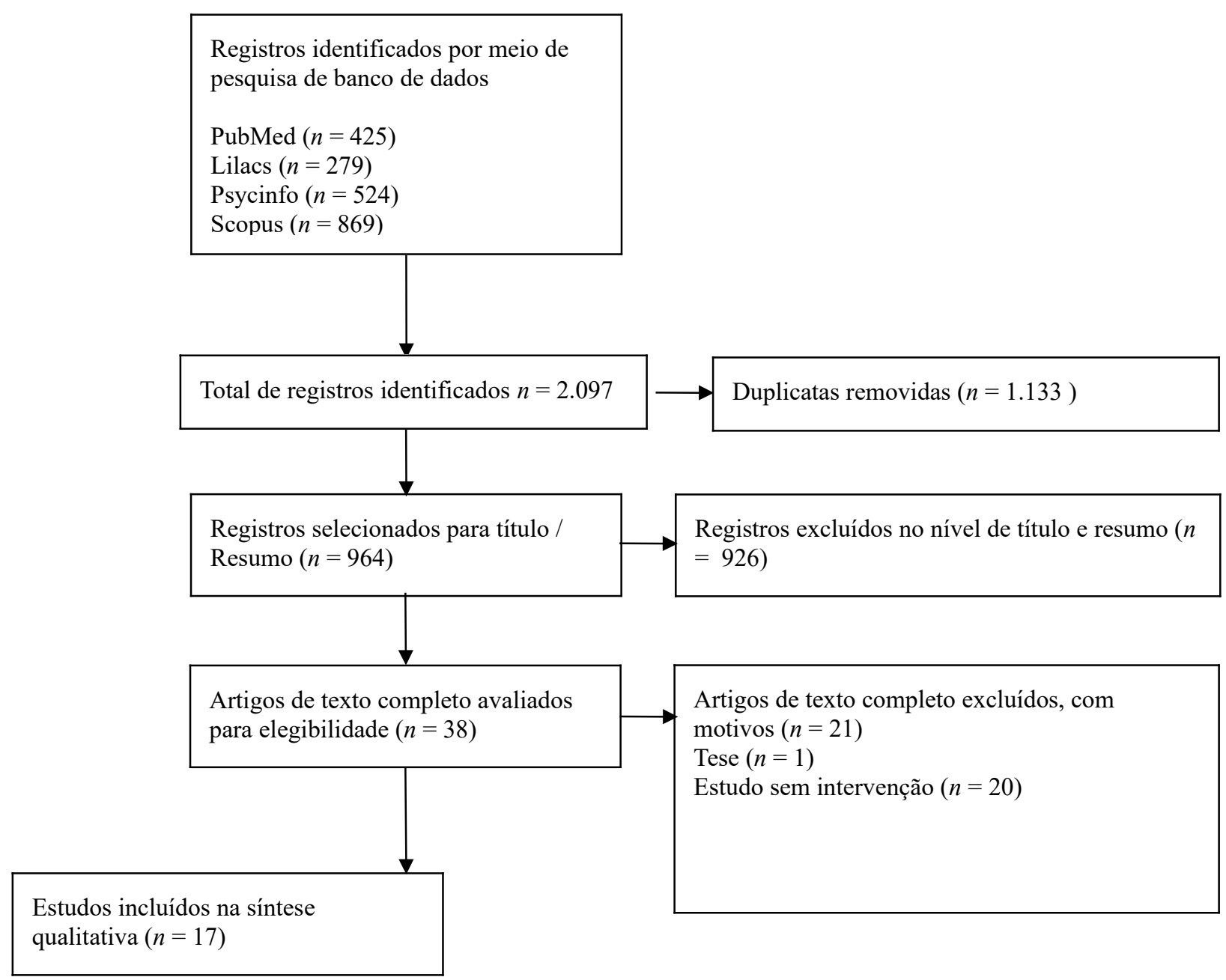

Figura 1. Fluxograma de estudos. 
Tabela 1

Características dos estudos incluídos

\begin{tabular}{|c|c|}
\hline Características & $\mathrm{N}$ de estudos \\
\hline \multicolumn{2}{|l|}{ Desenho de estudo } \\
\hline Ensaio clínico de controle randomizado & 3 \\
\hline Quase-experimental & 3 \\
\hline Estudo Experimental pré e pós-teste & 5 \\
\hline Estudo Pre e pós-teste & 2 \\
\hline Estudo de controle pareado de acompanhamento. & 1 \\
\hline Métodos mistos clínico/comunidade & 1 \\
\hline Estudo longitudinal pré e pós teste & 1 \\
\hline Estudo longitudinal quase-experimental & 1 \\
\hline \multicolumn{2}{|l|}{ Característica da população } \\
\hline Adolescentes em risco social (11 a 19 anos) & 5 \\
\hline Adolescentes em uso de substâncias (13 a 20 anos) & 4 \\
\hline Adolescentes em ansiedade social (14 a 15 anos) & 1 \\
\hline Adolescentes em dif. relacionamento social (13 a 17 anos) & 2 \\
\hline Adolescentes em transtornos alimentares ( 13 e 18 anos) & 1 \\
\hline Adolescentes de 10 a 16 anos & 2 \\
\hline Adolescentes em delinquência juvenil (12 a 18 anos) & 2 \\
\hline \multirow{2}{*}{\multicolumn{2}{|c|}{$\begin{array}{l}\text { Característica da intervenção } \\
\text { Área }\end{array}$}} \\
\hline & \\
\hline Assistência social & 3 \\
\hline Saúde & 5 \\
\hline Educação & 7 \\
\hline Segurança pública & 2 \\
\hline \multicolumn{2}{|l|}{ Estratégias } \\
\hline Instruções e atividades semanais & 7 \\
\hline Sessões grupais com interação social & 7 \\
\hline Instruções & 2 \\
\hline Sistema mobile coach & 1 \\
\hline
\end{tabular}

nerabilidade risco social compôs a temática de 5 artigos, seguida de uso de substâncias ( $n=$ 4), dificuldade de relacionamento social ( $n=$ 2), delinquência juvenil ( $n=2)$, ansiedade social $(n=1)$ e transtornos alimentares $(n=1)$. De acordo com a área de intervenção em que os estudos foram realizados, a maioria deles ( $n$ = 7) foi desenvolvida na educação, seguida de saúde $(n=5)$, assistência social $(n=3)$ e segurança pública $(n=2)$.

No que diz respeito as características das intervenções em competências sociais, a maioria dos estudos apresentados focalizou em estratégias de Instruções e actividades semanais ( $n$ = 7) (Campbell-Heider, Tuttle, \& Knapp, 2009; Eslami et al., 2015; Kimber \& Sandell, 2009; Lá- zaro, 2011; Melnyket al., 2013; van der Stouwe et al., 2018); seguido de instruções e grupos com interação social ( $n=7$ ) (Glenn et al., 2019; Harrell, Mercer. \& DeRosier, 2009; Leme, Fernandes, Jovarini, Achkar, \& Del Prette, 2016; Murta et al., 2016; Ramírez, Saldaña, Ardila, \& Meza, 2009; Roberts et al., 2011; Silva \& Murta, 2009); somente instruções $(n=2)$ (Babakhani, 2011; Melo, Pereira \& Silvério, 2014) e sistema mobile coach ( $n=1$ ) (Haug et al., 2017). Em relação à qualidade dos estudos, nove estudos foram de baixa qualidade, $\leq 75 \%$ segundo a lista de verificação de avaliação do EBL (Glynn, 2006) (Tabela 2). Estudos não foram excluídos com base na qualidade. Em média, a qualidade geral dos estudos incluídos foi de $73 \%$. 
ca garantir a aplicação dos temas abordados no contexto dos participantes para a manutenção dos comportamentos aprendidos.

Foram encontrados quatro estudos que desenvolveram intervenções com um tempo superior a seis meses, através de encontros e actividades semanais. Grande parte dos estudos foram desenvolvidos durante um período superior a dois meses de duração. Quatro estudos realizaram avaliação de seguimento de um a doze meses após a intervenção. Esses critérios auxiliam no fortalecimento e generalização das atitudes positivas treinadas durante as intervenções.

Os estudos com relação ao desenvolvimento de atitudes positivas desenvolvidas pelos adolescentes através da intervenção instruções e actividades semanais, demonstraram Walta qualidade nos resultados, apresentando um aumento das competências sociais (assertividade, auto-controle, cooperação, empatia) redução de comportamento problema (Eslami et al., 2015); Redução no uso de álcool e drogas (Kimber \& Sandell, 2009); Impacto positivo na actividade física, no IMC, nos desfechos psicossociais e no desempenho de grau escolar (Melnyk et al., 2013); Melhoria de atribuição de intenções hostis a juvenis como empatia afectiva e diminuição de distorções cognitiva (van der Stouwe et al., 2018). Nos demais estudos os resultados da intervenção em competências sociais resultou na melhora da auto-estima, percepção da aparência física, auto-conceito relacionado ao peso e forma, autoconceito relacionado aos outros, felicidade, satisfação percepção de status intelectual e escolar, liberdade de ansiedade timidez e popularidade
(Lázaro et al., 2011); Redução de problemas de saúde mental, saúde física, relação entre pares e agressão (Campbell-Heiderer et al., 2009).

Em contraste, um estudo não apresentou resultado em atitude positiva em sua intervenção, resultando na baixa relação de eficácia na prevenção de reincidência geral, na frequência, ou na gravidade da reincidência de delitos em adolescentes em um seguimento de 6 e 12 meses (van der Stouwe et al., 2018).

Instruções e grupo com interacção social. Programas desenvolvidos com instruções e grupos com interacção social utilizavam como estratégias a promoção e o desenvolvimento de vivências nos encontros de grupos de adolescentes, por meio de técnicas em competências sociais, cognitivo-comportamental e de aprendizagem social relacionadas aos temas abordados nos encontros, muitos com componente parental, proporcionaram, assim, a visualização e intervenção directamente no grupo através das relações interpessoais desenvolvidas no grupo (Tabela 4). Foram encontrados três estudos que desenvolveram intervenções com um tempo superior a seis meses com encontros semanais e grupos de interacção social. A maioria dos estudos foram desenvolvidos entre nove e doze encontros semanais. Apenas um estudo teve avaliação de seguimento com um seguimento de um e sete meses após a intervenção.

Os resultados de atitudes positivas a partir das intervenções em instruções e interacção social nos estudos analisados, foram, a maioria, desenvolvidos com baixa qualidade, apresentando resultados de intervenções em competências sociais o auto-conceito 
Tabela 2

Análise dos domínios de checklist de avaliação do EBL para o estudo incluído

\begin{tabular}{cccccc}
\hline & \multicolumn{5}{c}{ Validade (\%) } \\
\cline { 2 - 5 } Estudos & $\begin{array}{c}\text { Domínio } \\
\text { populacional }\end{array}$ & $\begin{array}{c}\text { Domínio de } \\
\text { coleta de } \\
\text { dados }\end{array}$ & $\begin{array}{c}\text { Domínio de } \\
\text { projeto de } \\
\text { estudo }\end{array}$ & $\begin{array}{c}\text { Domínio de } \\
\text { resultado }\end{array}$ & $\begin{array}{c}\text { Validade geral } \\
\text { do estudo (\%) }\end{array}$ \\
\hline Babakhani, 2011 & 55.55 & 75 & 80 & 66.66 & 67.85 \\
Campbell-Heideret al., 2009 & 55.55 & 66.66 & 80 & 66.66 & 67.85 \\
Eslami, et al., 2015 & 88.88 & 100 & 80 & 66.66 & 82.75 \\
Glenn, 2018 & 60 & 75 & 100 & 66.66 & 75 \\
Harrell, et al., 2009 & 44 & 100 & 80 & 50 & 67.85 \\
Haug, et al., 2017 & 100 & 85.71 & 100 & 66.66 & 88.88 \\
Kimber\&Sandell, 2009 & 66.66 & 75 & 100 & 66.66 & 75 \\
Lazaro, 2011 & 44.44 & 87.5 & 80 & 50 & 67.85 \\
Leme etal., 2016 & 50 & 100 & 80 & 50 & 72 \\
Melnyk et al., 2013 & 87.5 & 100 & 100 & 83.33 & 96.29 \\
Melo,Pereira \& Silvério, 2014 & 40 & 87.5 & 60 & 50 & 53.57 \\
Murta\&cols, 2016 & 44.44 & 87.5 & 80 & 80 & 67.85 \\
Ramirez et al., 2009 & 40 & 37.5 & 50 & 33.33 & 41.66 \\
Roberts et al., 2011 & 100 & 100 & 100 & 66.66 & 92.85 \\
Silva \& Murta, 2009 & 50 & 75 & 60 & 50 & 60 \\
Stouwe, 2018. & 77.77 & 87.5 & 60 & 66.66 & 75 \\
Stouwe, et al., 2018 & 100 & 100 & 75 & 66.66 & 88.88 \\
\hline
\end{tabular}

global aprimorado, auto-eficácia social aumentada e diminuição dos problemas internalizantes (Harrell et al., 2009); aumento da empatia, auto-controle, assertividade, abordagem afetiva, desenvoltura social, habilidades globais em auto-eficácia e percepção de apoio social de professores (Leme et al., 2016); redução de atitudes de endosso a papéis de género masculino com restrição emocional, aumento em intenção de respostas assertivas na condição de vitimização, redução em intenções de respostas violentas na condição de perpetração e prática de competências sociais de autocontrole e expressão das emoções (Murta et al., 2016); aumento das competências sociais (defesa assertiva de direitos pessoais, expressão de reclamações responsáveis, escuta atenta, respostas efectivas e críticas) (Ramírez et al., 2009); comportamento de "expressar carinho", "fazer elogios", "pedir ajuda", "dizer não" e "puxar conversa" (Silva \& Murta, 2009). Dois estudos demonstraram alta qualidade de evidência, apresentando como resultado de atitudes positivas a probabilidade reduzida de uso de álcool e tabaco (Roberts et al., 2011) e menor ansiedade social em contextos estruturados (Glenn et al., 2019). 
Instruções. Intervenções baseadas em instruções buscam fornecer informações através de encontros de grupo abordando temas relacionados a competências sociais, saúde e teoria cognitivo comportamental. Os estudos que utilizaram somente instruções foram desenvolvidos em seis e doze sessões e não apresentaram avaliação de seguimento (Tabela 5). Os dois estudos demonstraram baixa qualidade nos resultados de atitudes positivas com desfechos de diminuição da agressão verbal (Barbakhani, 2011) e aumento de níveis de conhecimento sobre sexualidade, assertividade e competências sociais (Silvério, 2014).

Sistema mobile coach. O uso da tecnologia como estratégia em programas de competências sociais é bastante inovador (Tabela 6). 0 sistema mobile coach é uma ferramenta desenvolvida a partir de um estudo com actividades de auto gestão em competências sociais, resistência ao uso de substâncias, feedback de mensagens e textos, incentivos e recompensas virtuais. O programa teve uma duração de seis meses com mais seis meses de avaliação de seguimento e apresentou alta qualidade nos resultados. Como desfecho de atitudes positivas o estudo apresentou diminuição do stress percebido, aumento nas competências gerais de vida e diminuição de uso de álcool (Haug et al., 2017).

\section{Discussões}

A revisão apresenta estudos que analisam intervenções em competências sociais com adolescentes e o desenvolvimento de atitudes positivas em situação de vulnerabilidade.
Dezassete estudos foram revistos sistematicamente para abordar a temática acima.

\section{Instruções e actividades semanais}

A estratégia de instruções e actividades semanais apresentou alta qualidade dos estudos, demonstrando como resultado o aumento das competências sociais, assertividade, auto controle, cooperação, empatia, redução de comportamento problema (Eslami et al., 2015), redução no uso de álcool e drogas (Kimber \& Sandell, 2009), impacto positivo na actividade física, no IMC, nos desfechos psicossociais e no desempenho de grau escolar (Melnyk et al., 2013), melhoria de atribuição de intenções hostis a juvenis com empatia afectiva e diminuição de distorções cognitiva (van der Stouwe et al., 2018).

Estudos realizados com estratégia semelhante demonstram a diminuição de comportamentos problema. Alguns autores identificaram a eficácia de programas de competências sociais na resistência ao uso de álcool e drogas (Kimber \& Sandell, 2009). Outros estudos apontaram a eficiência de intervenções semelhantes na prevenção de comportamentos de agressão e delinquência (Botvin \& Griffin, 2014).

Algumas características das intervenções em competências sociais apontadas por Kimber e Sandell (2009) em seu estudo para aumentar a eficácia das variáveis nos resultados. Esse autor indicou que, o tempo de duração dos programas, a inclusão de vários componentes na avaliação e resultado, uma gama de graus escolares e consequente variabilidade de idade da amostra bem como a adopção da eficácia de participantes em contrapartida a uma 


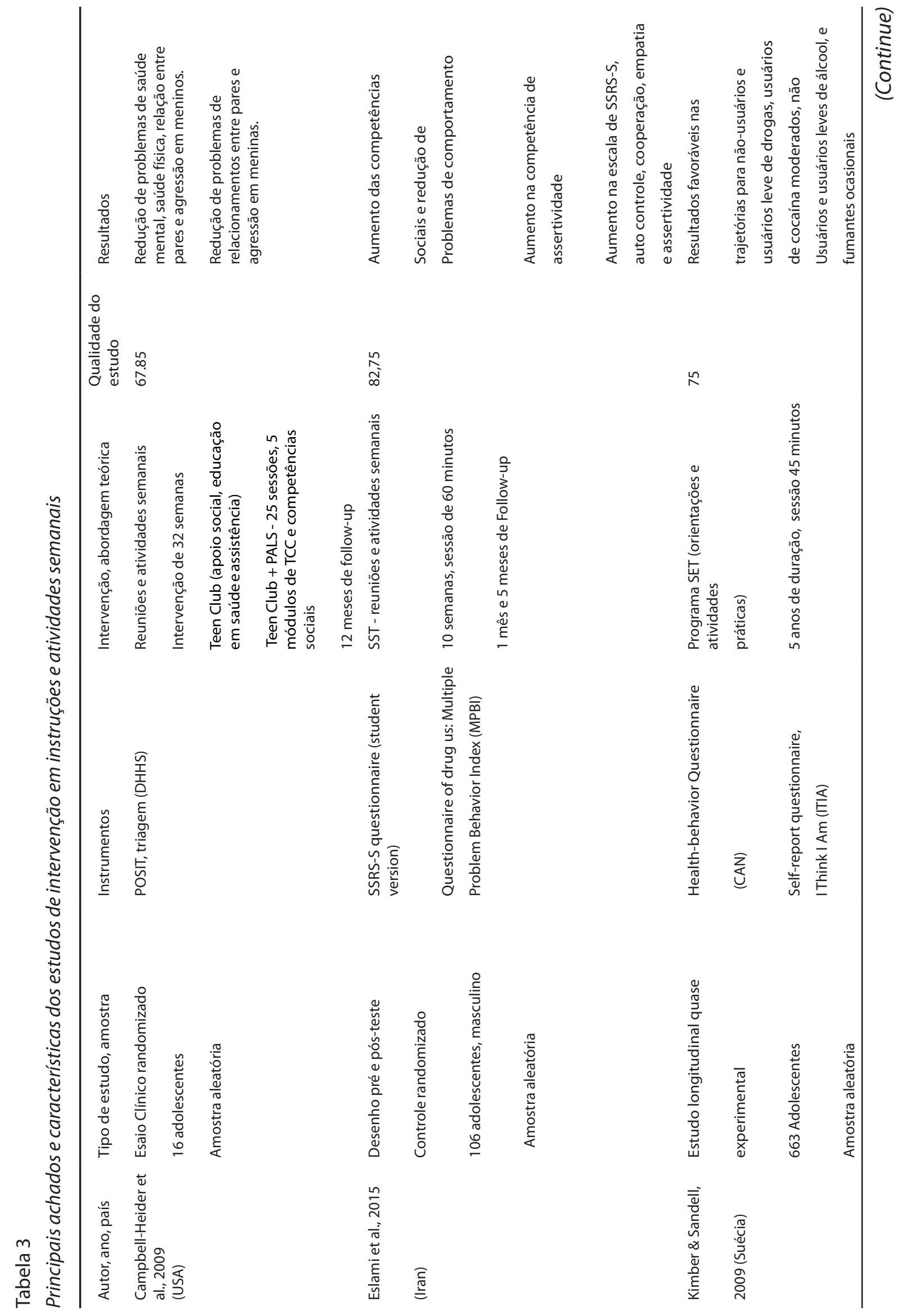




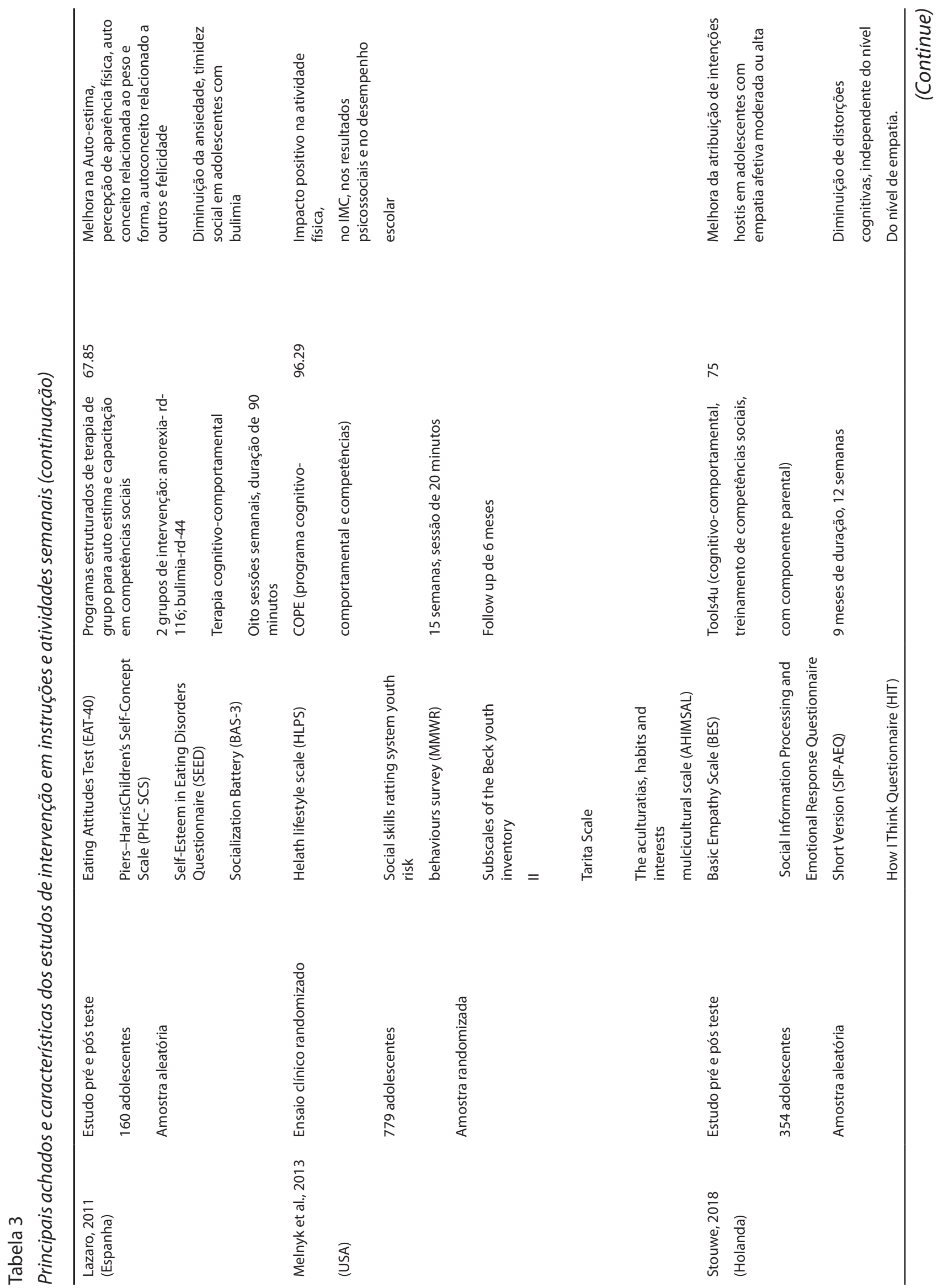




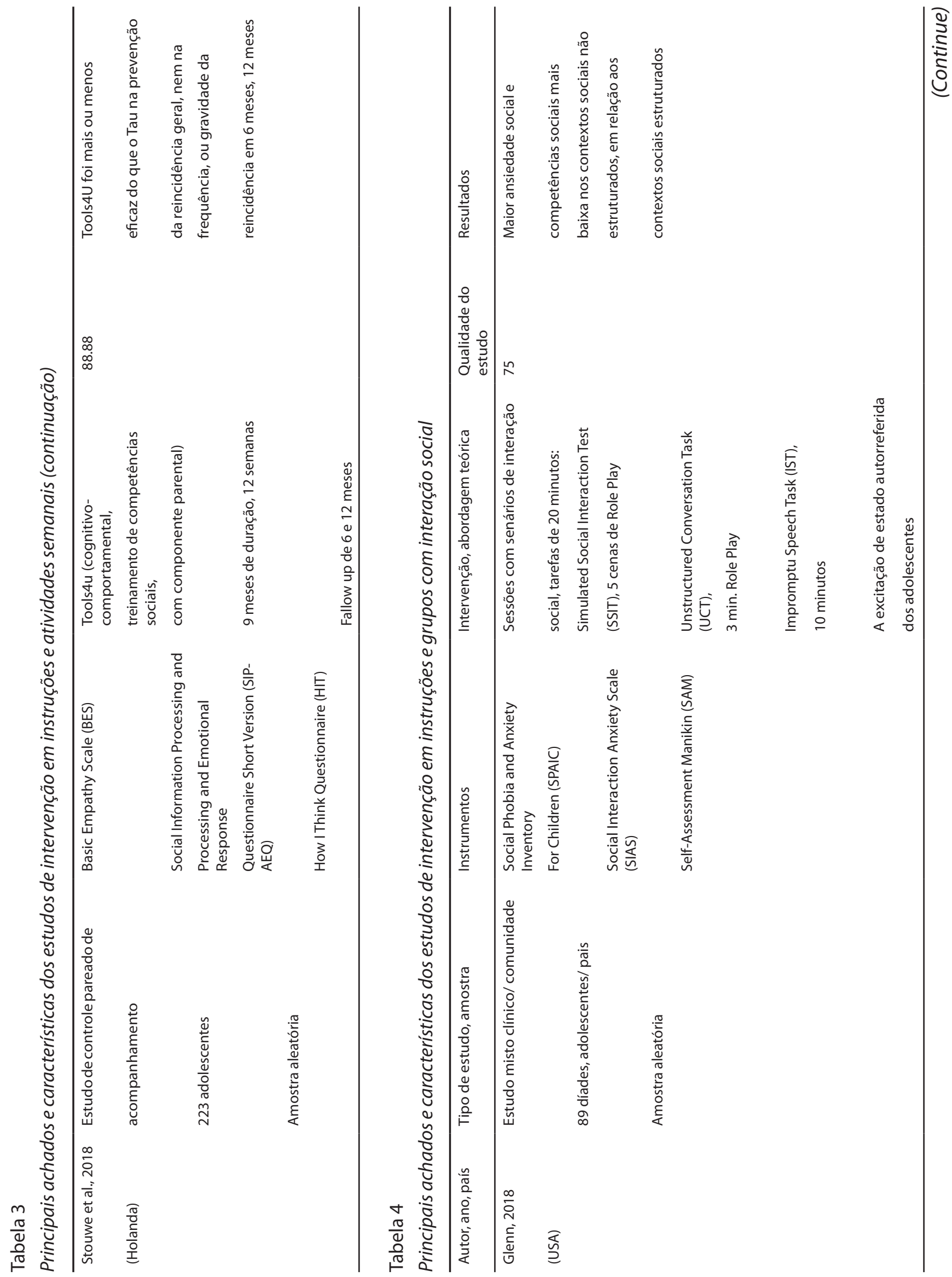



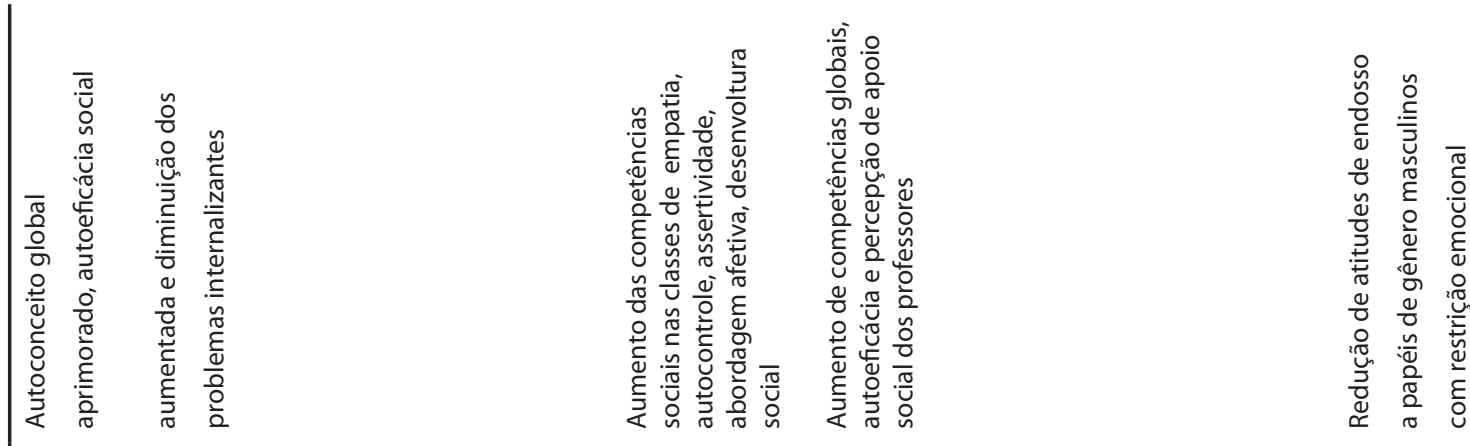

๖ำ

:

N

$\stackrel{\substack{\infty \\ 6}}{6}$

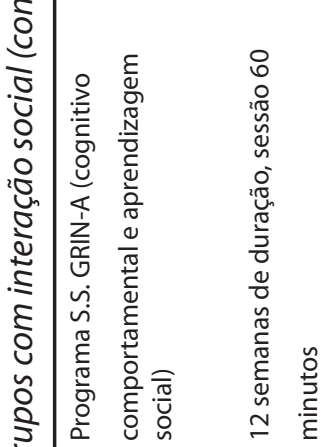

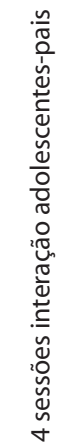

$\stackrel{n}{\frac{N}{5}}$
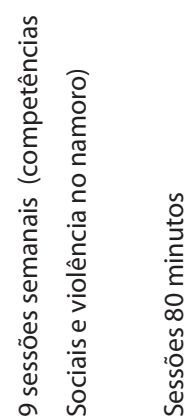

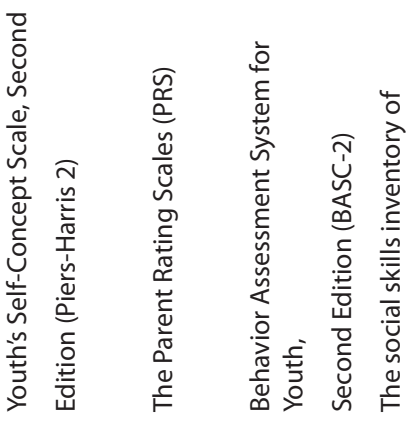

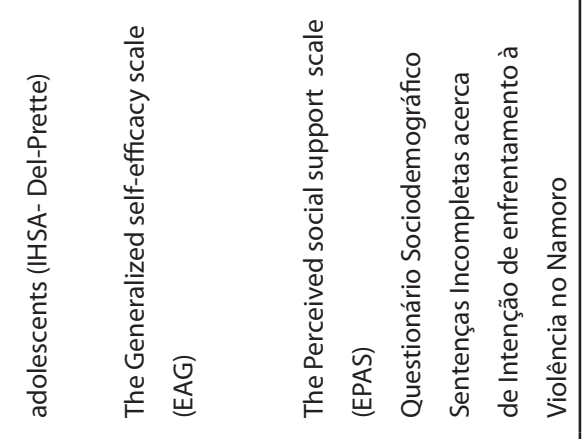




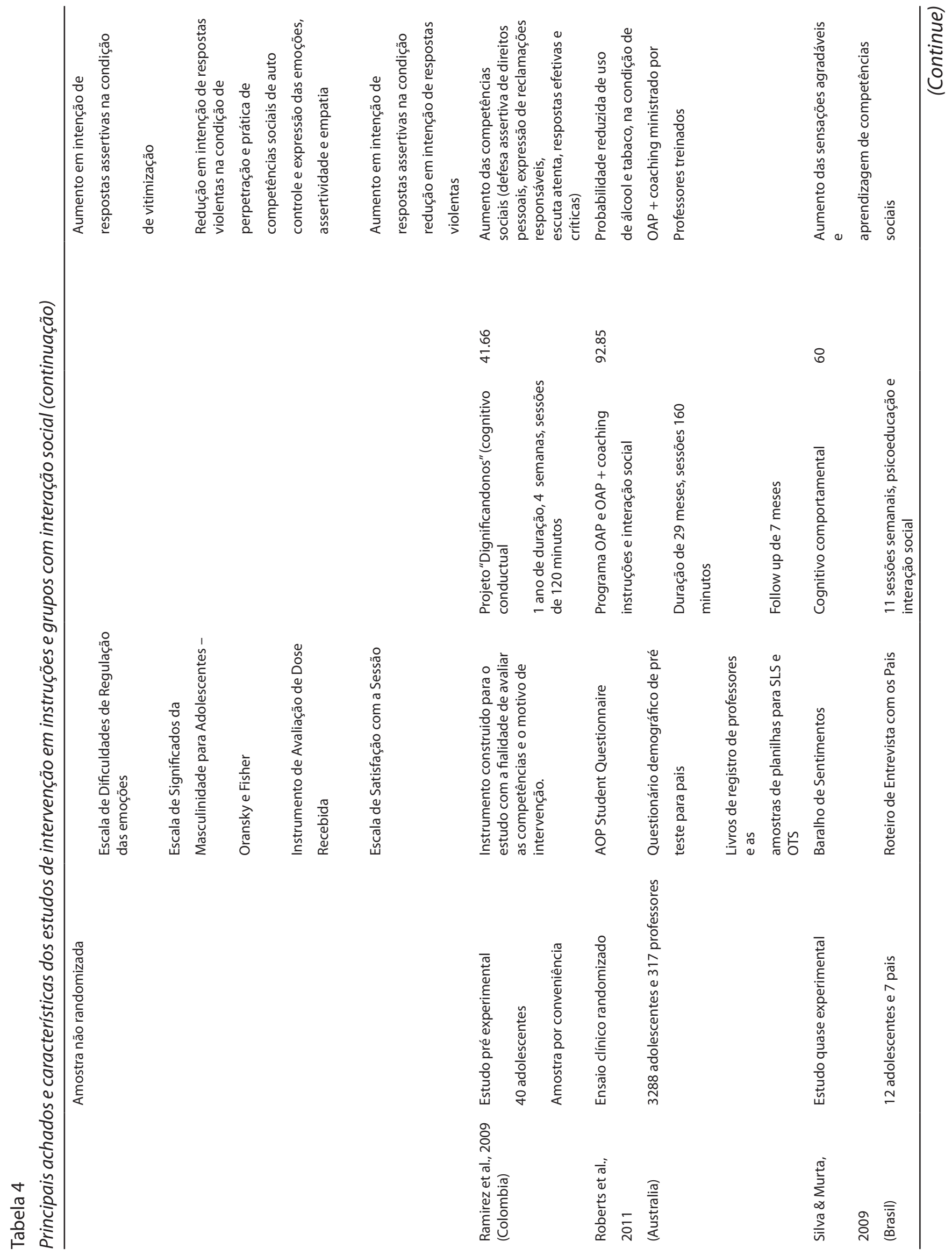




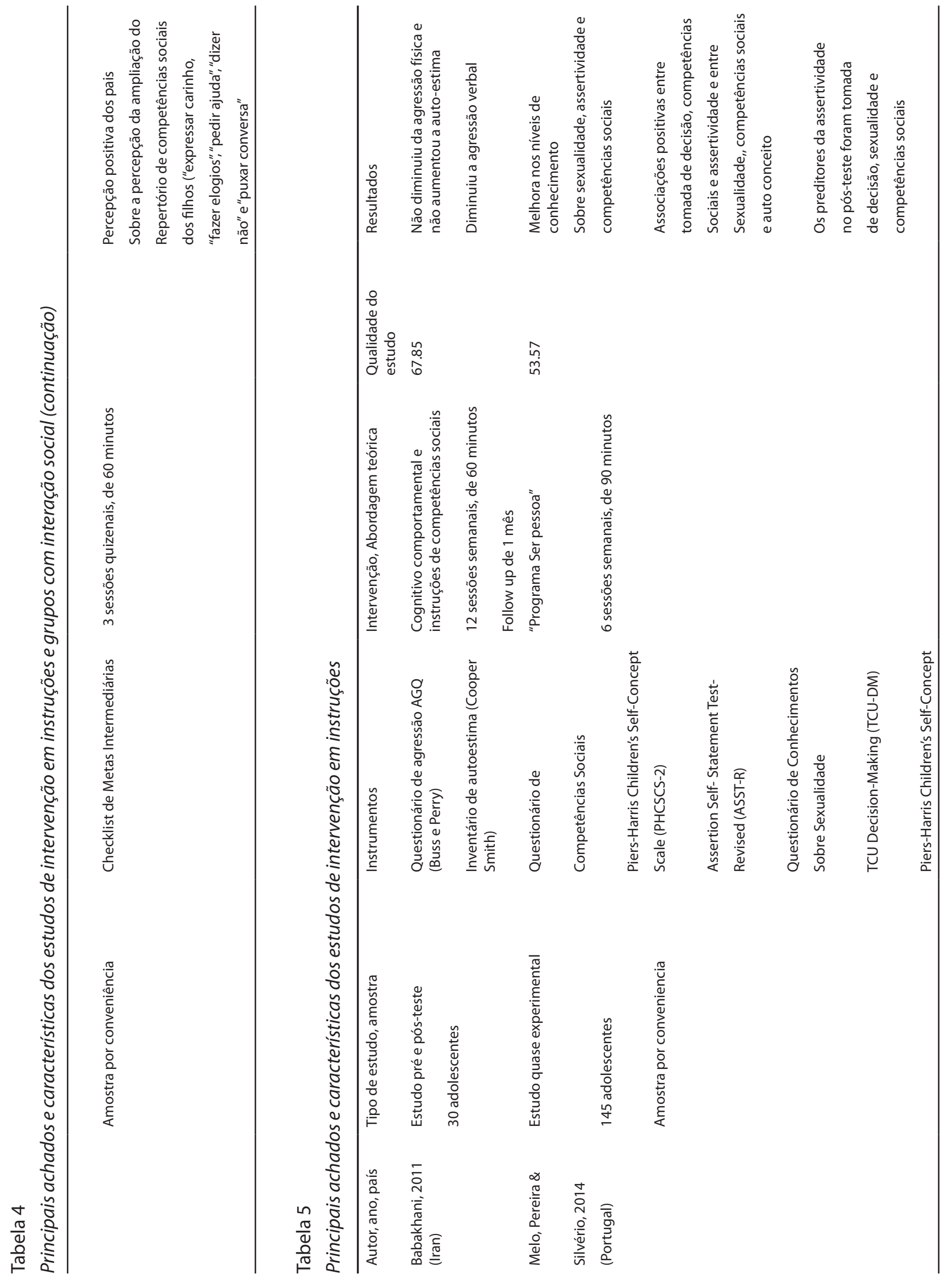




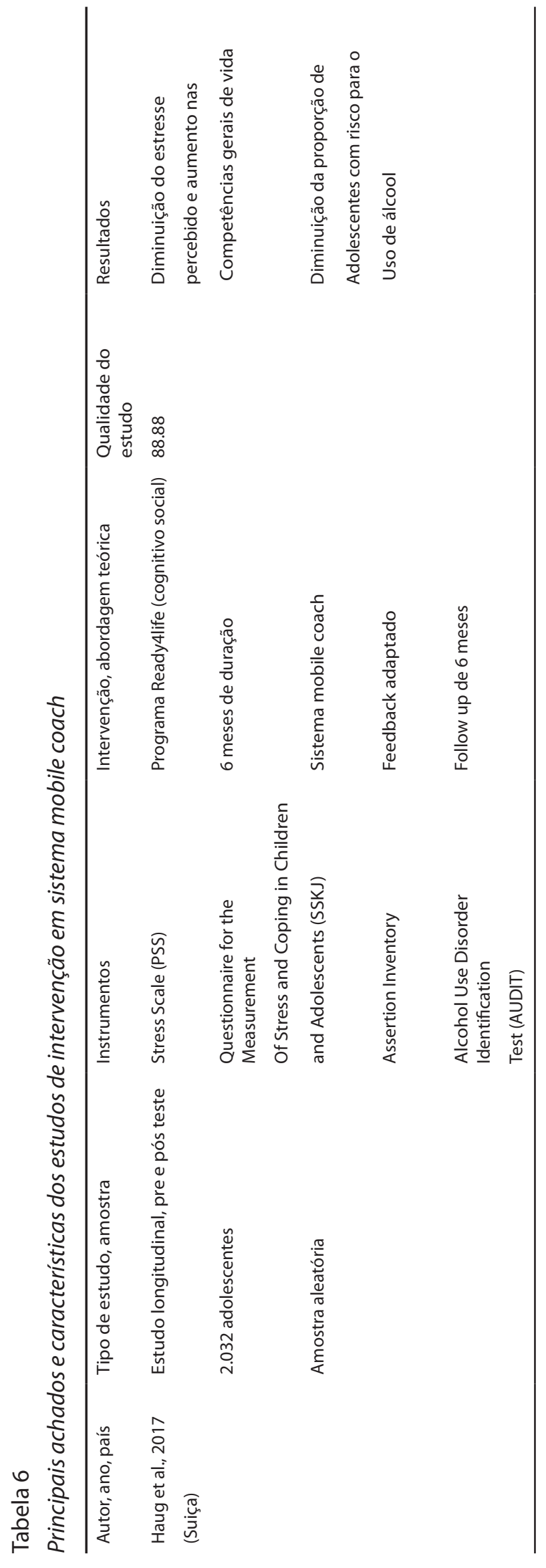


eficácia clínica, foram indicadores de maior eficácia em programas de competências sociais para adolescentes na escola. Além das características apontadas acima outros estudos identificaram resultados positivos em programas de multicomponentes a curto prazo em adolescentes. Esses programas utilizaram uma estratégia como instruções em saúde, medidas frequentes de IMC e acompanhamento semanal de nutricionista, orientações e práticas de actividade física e técnicas de modificação de comportamento (Bonsergent et al., 2013).

\section{Instruções e grupo com interacção social}

Os estudos desenvolvidos a partir da estratégia de instruções e grupo de interacção social foram a maioria avaliados com baixa qualidade de resultados. Os estudos que demonstraram alto poder conclusivo, apresentaram como resultado de atitudes positivas a probabilidade reduzida de uso de álcool e tabaco (Roberts et al., 2011) e menor ansiedade social em contextos estruturados (Glenn et al., 2019). Entre as limitações apontadas pelos autores nos estudos encontram-se o tamanho da amostra, o delineamento do estudo, falta de controlo de variáveis, processo de avaliação das competências sociais, o tempo de duração e seguimento para a detecção de efeitos do programa.

Como limitação nas intervenções baseadas em instruções e grupo com interacção social identificou-se o viés dos observadores ou da equipe de intervenção na possibilidade de variabilidade na coordenação dos encontros de grupo e actividades de interacção com os adolescentes, apontando como características dessa limitação a construção do processo de formação dos educadores e a idade dos mes- mos, pois se tratando de uma relação de interacção e sendo a maioria adultos, a sugestão dos autores para facilitação das interacções pelos adolescentes seriam intervenções através de adolescentes da mesma idade, ou seja, relação de pares (Karp et al., 2018).

A eficácia em programas de intervenção em competências sociais no ensino médio foi questionada por alguns investigadores, pois, a influência de pares no ambiente escolar na aprendizagem de comportamentos é bastante relevante. $\mathrm{O}$ tempo de convivência com os amigos e os meios de comunicação geraram modelos de identificação com um poder maior que algumas intervenções em competências sociais na vida dos adolescentes (Botvin \& Griffin, 2014). Porém, em uma revisão de programas de prevenção de uso de substâncias desenvolvidos na escola identificou a efectividade de estratégias de ensino de competências sociais e interacção social, pois permitiram ao adolescente desenvolver uma avaliação crítica de normas e atitudes para competências de resistência ao uso precoce de substâncias. Outro estudo com foco nos programas de intervenção em interações sociais apresentou efeitos positivos duradouros em comportamentos de resistência ao uso de substâncias em adolescentes até o final do ensino médio (Botvin \& Griffin, 2014). Em relação à eficácia de intervenções em competências sociais os resultados apontaram para a condução de programas que combinem estratégias de instrução e técnicas de modelagem comportamental, técnicas de auto-regulação e habilidades de resolução de problemas (Marsch \& Borodovsky, 2016). 


\section{Instruções}

As intervenções baseadas somente em instruções demonstraram baixa qualidade em seus resultados para desenvolvimento de atitudes positivas. Esses estudos apresentaram como limitações o tempo de duração do programa, o tamanho da amostra e a não realização de avaliação de seguimento (Barbakhani, 2011; Silvério, 2014). Nessa direcção, os questionamentos são dirigidos aos programas que utilizaram somente a estratégia de informação para treino em competências sociais, pois as mesmas não apresentaram eficácia para o desenvolvimento de atitudes positivas em adolescentes (Clarey, Hokoda, \& Ulloa, 2010). Na mesma direcção, as classes de comportamento como assertividade, ansiedade, agressividade e delinquência não apresentaram desfecho positivo na utilização de tal estratégia (Kimber, 2009), porém intervenções por meio de instruções em competências sociais em factores como uso de álcool e drogas e comportamento sexual de risco demonstraram resultados positivos (Botvin \& Griffin, 2014)

\section{Sistema mobile coach}

A estratégia de sistema mobile coach apresentou alta qualidade nos resultados em atitudes positivas como diminuição do stress percebido, aumento nas competências gerais de vida e diminuição de uso de álcool. Algumas limitações levantadas pelos autores foram a restrição da eficácia a adolescentes sem uso regular de cigarro e a perda de participantes na avaliação de seguimento (Haug et al., 2017). Estudos sobre o potencial e a eficácia do uso de ferramentas electrónicas em intervenções precoces em adolescentes para a diminuição do uso de álcool e drogas já encontram-se em desenvolvimento (Marsch \& Borodovsky, 2016).

Programas gerais de competências sociais e de vida para prevenção do uso de substâncias desenvolvidos internacionalmente (Botvin \& Griffin, 2014) combinam, regularmente, no treino de competências a autogestão, competências sociais (auto consciência, estratégia de enfrentamento, competências de comunicação e assertividade) e competências de resistência ao uso de substâncias. Embora tais intervenções apresentarem alta eficácia na prevenção ou diminuição do uso problemático das substâncias (Faggiano, 2014) a implementação no ambiente escolar apresentaram diferentes obstáculos como aplicadores sem motivação, conhecimento e competências, disponibilidade e viabilidade económica para desenvolver o programa (Ellickson, 2014).

Esses obstáculos que poderiam inviabilizar a entrega dos programas e consequente a disseminação do treino de competências sociais nas escolas podem ser superados por intervenções electrónicas (Haug et al., 2017). Essa intervenção com capacidade de um alcance amplo de instituições e participantes a baixo custo e apresentaram o conteúdo e resultados automaticamente disponíveis aos participantes, além da atractividade das ferramentas online para os adolescentes, favorecendo assim a adesão e a privacidade dos mesmos (Kuntsche \& Labhart, 2014).

\section{Pontos fortes e limitações}

Esta revisão foi realizada seguindo a lista de verificação recomendada pela PRISMA (Moher et al., 2015). Todas as etapas implementadas na 
estratégia de pesquisa para cada banco de dados foram amplamente relatadas. Nos estudos incluídos, a heterogeneidade esteve presente, principalmente devido a questões envolvendo as condições de vulnerabilidade das populações do estudo, intervenções e desfechos.

\section{Conclusão}

As intervenções em competências sociais com adolescentes em situação de vulnerabilidade podem ser uma estratégia eficaz para o desenvolvimento de atitudes positivas, principalmente as estratégias de instruções e actividades semanais e o sistema mobile coach que apresentaram resultados positivos nos estudos analisados. Estes resultados fornecem evidências para pesquisadores, profissionais e instituições para a prática em prevenção e promoção a saúde de adolescentes.

\section{Pontos chave}

Estratégias baseadas somente em instruções, nem sempre são eficazes para desenvolver atitudes positivas em adolescentes em condições de vulnerabilidade, pois apresentam baixa qualidade de resultados.

Instruções e grupo com interacção social podem ser uma intervenção eficaz para desenvolvimento de atitudes como redução do uso de álcool e drogas e ansiedade social, embora a maioria dos estudos apresentem conclusões fracas;

O sistema mobile coach foi efectivo com alta qualidade dos resultados no desenvolvimento de atitudes como na diminuição de stress, aumento de competências gerais de vida e redução do uso de álcool em adolescentes;

Programas desenvolvidos com instruções e actividades semanais, foram mais eficazes para o desenvolvimento de atitudes positivas em adolescentes como aumento das competências sociais, assertividade, auto-controle, cooperação, empatia, redução de comportamento problema, redução do uso de drogas, actividade física, IMC, desenvolvimento psicossocial, desempenho escolar, atribuição de intenções hostis e diminuição de distorções cognitivas em adolescentes.

\section{Referências}

Babakhani, N. (2011). The effects of social skills training on self-esteem and aggression male adolescents. Procedia-Social and Behavioral Sciences, 30, 1565-1570. https:// doi.org/10.1016/j.sbspro.2011.10.304

Beauchamp, M. H., \& Anderson, V. (2010). SOCIAL: An integrative framework for the development of social skills. Psychological Bulletin, 136, 39-64. https://doi.org/10.1037/ a0017768

Bonsergent, E., Agrinier, N., Thilly, N., Tessier, S., Legrand, K., Lecomte, E.,... \& PRALIMAP Trial Group. (2013). Overweight and obesity prevention for adolescents: a cluster randomized controlled trial in a school setting. American Journal of Preventive Medicine, 44, 30-39. https://doi. org/10.1016/j.amepre.2012.09.055

Botvin, G.J., \& Griffin, K.W. (2014), Life skills training: preventing substance misuse by enhancing individual and social competence. In K. Weichold \& F. Giannotta F. (eds.), Theory-based approaches to substance misuse and abuse prevention in school: New directions for youth development (pp- 5766). San Francisco: Jossey-Bass.

Campbell-Heider, N., Tuttle, J., \& Knapp, T. R. (2009). CE FEATURE: The effect of positive adolescent life skills training on long term outcomes for high-risk teens. Journal of Addictions Nursing, 20, 6-15. https://doi. org/10.1080/10884600802693165

Clarey, A., Hokoda, A., \& Ulloa, E. C. (2010). Anger control and acceptance of violence as mediators in the relationship between 
exposure to interparental conflict and dating violence perpetration in Mexican adolescents. Journal of Family Violence, 25, 619-625. https://doi.org/10.1007/s10896010-9315-7

Ellickson, P.L. (2014), You've shown the program is effective. Now what? In K. Weichold, \& F. Giannotta (eds), Theory-based approaches to substance misuse and abuse prevention in school: New directions for youth development (pp. 95-106). San Francisco: Jossey-Bass.

Eslami, A. A., Ghofranipour, F., Bonab, B. G., Zadeh, D. S., Shokravi, F. A., \& Tabatabaie, M. G. (2015). Evaluation of a schoolbased educational program to prevent adolescents' problem behaviors. Journal of Education and Health Promotion, 4, 30-53. https://doi.org/10.4103/2277-9531.154127

Faggiano, F., Minozzi, S., Versino, E., \& Buscemi, D. (2014). Universal school-based prevention for illicit drug use. Cochrane Database Systematic Reviews, 12, CD003020. https:// doi.org/10.1002/14651858.CD003020.pub3

Glenn, L. E., Keeley, L. M., Szollos, S., Okuno, H., Wang, X., Rausch, E.,... \& Augenstein, T. M. (2019). Trained observers' ratings of adolescents' social anxiety and social skills within controlled, cross-contextual social interactions with unfamiliar peer confederates. Journal of Psychopathology and Behavioral Assessment, 41, 1-15. https:// doi.org/10.1007/s10862-018-9676-4

Glynn, L. (2006). A critical appraisal tool for library and information research. Library $\mathrm{Hi}$ Tech, 24, 387-399. Recuperado de https:// www.emerald.com/insight/publication/ issn/0737-8831

Harrell, A. W., Mercer, S. H., \& DeRosier, M. E. (2009). Improving the social-behavioral adjustmentofadolescents:Theeffectiveness of a social skills group intervention. Journal of Child and Family Studies, 18, 378-387. https://doi.org/10.1007/s10826-008-9241-y

Haug, S., Castro, R. P., Meyer, C., Filler, A., Kowatsch, T., \& Schaub, M. P. (2017). A mobile phone-based life skills training program for substance use prevention among adolescents: pre-post study on the acceptance and potential effectiveness of the program, Ready4life. JMIR mHealth and uHealth, 5, e143. Recuperado de https:// mhealth.jmir.org/2017/10/e143/pdf

Karp, J. N., Makol, B. A., Keeley, L. M., Qasmieh, N., Deros, D. E., Weeks, J. W.,... \& De Los Reyes, A. (2018). Convergent, incremental, and criterion-related validity of multi informant assessments of adolescents' fears of negative and positive evaluation. Clinical Psychology \& Psychotherapy, 25, 217-230. https://doi.org/10.1002/cpp.2156

Kimber, B., \& Sandell, R. (2009). Prevention of substance use among adolescents through social and emotional training in school: A latent-class analysis of a fiveyear intervention in Sweden. Journal of Adolescence, 32, 1403-1413. https://doi. org/10.1016/j.adolescence.2009.10.005

Kuntsche, E., \& Labhart, F. (2014). The future is now-using personal cellphones to gather data on substance use and related factors. Addiction, 109, 1052-1053. https://doi. org/10.1111/add.12540

Lázaro, L., Font, E., Moreno, E., Calvo, R., Vila, M., Andrés-Perpiñá, S., ... \& Castro-Fornieles, J. (2011). Effectiveness of self esteem and social skills group therapy in adolescent eating disorder patients attending a day hospital treatment programme. European Eating Disorders Review, 19, 398-406. https:// doi.org/10.1002/erv.1054

Leme, V. B. R., Fernandes, L. D. M., Jovarini, N. V., Achkar, A. M. E., \& Del Prette, Z. A. P. (2016). Social Skills Program for adolescents in vulnerable social contexts. Psico-USF, 21, 595-608. http://dx.doi.org/10.1590/141382712016210313

Lipsey, M. W., Howell, J. C., Kelly, M. R., Chapman, G., \& Carver, D. (2010). Improving the effectiveness of juvenile justice programs: $A$ new perspective on evidence-based practice. Washington, DC: Georgetown University, Centre for Juvenile Justice.

Marsch, L. A., \& Borodovsky, J. T. (2016). Technology-based interventions for preventing and treating substance use among youth. Child and Adolescent Psychiatric Clinics, 25, 755-768. https://doi. org/10.1016/j.chc.2016.06.005 
Matos, M. G., Gaspar, T., Ferreira, M., Tomé, G., Camacho, I., Reis, M., \& Equipa Aventura Social (2012). Keeping a focus on selfregulation and competence: "find your own style", A school based program targeting at risk adolescents. Journal of Cognitive and Behavioral Psychotherapies, 12, 39-48. Recuperado de http://jebp.psychotherapy. ro/vol-xii-no-1-2012/keeping-focusregulation-competence-find-style-schoolbased-program-targeting-risk-adolescents/ Melo, M., Pereira, M. G., \& Silvério, J. (2014). Impacto de um Programa de Competências em Alunos do $2^{\circ}$ Ciclo de Escolaridade. Psicologia Escolar e Educacional, 18, 113-124. Recuperado de https://www.redalyc.org/ pdf/2823/282330520012.pdf

Melnyk, B. M., Jacobson, D., Kelly, S., Belyea, M., Shaibi, G., Small, L.,... \& Marsiglia, F. F. (2013). Promoting healthy lifestyles in high school adolescents: a randomized controlled trial. American Journal of Preventive Medicine, 45, 407-415. https:// doi.org/10.1016/j.amepre.2013.05.013

Moher, D., Shamseer, L., Clarke, M., Ghersi, D., Liberati, A., Petticrew, M.,... \& Stewart, L. A. (2015). Preferred reporting items for systematic review and metaanalysis protocols (PRISMA-P) 2015 statement. Systematic Reviews, 4, 1-9. https://doi.org/10.1186/2046-4053-4-1

Murta, S. G., Moore, R. A., Miranda, A. A. V., Cangussú, E. D. A., dos Santos, K. B., Bezerra, K. L. T., \& Veras, L. G. (2016). Efeitos de um Programa de Prevenção à Violência no Namoro. Psico-USF, 21, 381-393. http:// dx.doi.org/10.1590/1413-82712016210214

Ramírez, L. Y. Á., Saldaña, C., Ardila, J. A. M., \& Meza, V. P. (2009). Asertividad, escucha y afrontamiento de la crítica en adolescentes ensituacióndecalle. RevistaLatinoamericana de Ciencias Sociales, Niñez y Juventud, 7, 1535-1543. Recuperado de https://www. redalyc.org/pdf/773/77314999015.pdf

Roberts, C., Williams, R., Kane, R., Pintabona, Y., Cross, D., Zubrick, S., \& Silburn, S. (2011). Impact of a mental health promotion program on substance use in young adolescents. Advances in Mental
Health, 10, 72-82. https://doi.org/10.5172/ jamh.2011.10.1.72

Silva, M., \& Murta, S. G. (2009). Treinamento de habilidades sociais para adolescentes: uma experiência no programa de atenção integral à família (PAIF). Psicologia: Reflexão e Crítica, 22, 136-143. http://dx.doi. org/10.1590/S0102-79722009000100018

Silvério, M. (2014). Developmentally appropriate sexualityeducation theory, conceptualization, and practice. Unpublished doctoral dissertation. Berkeley, CA: University of California.

van der Stouwe, T., Asscher, J. J., Hoeve, M., van der Laan, P. H., \& Stams, G. J. J. (2018). Social skills training (SST) effects on social information processing skills in justiceinvolved adolescents: Affective empathy as predictor or moderator. Children and Youth Services Review, 90, 1-7. https://doi. org/10.1016/j.childyouth.2018.05.006 